International Journal of Applied Mathematics

Volume 31 No. $2 \quad 2018,263-277$

ISSN: 1311-1728 (printed version); ISSN: 1314-8060 (on-line version)

doi: http://dx.doi.org/10.12732/ijam.v31i2.8

\title{
ON THE WEAK SOLUTIONS OF THE URYSOHN-STIELTJES FUNCTIONAL INTEGRAL EQUATIONS
}

\author{
Ahmed M. El-Sayed ${ }^{1}$, Masouda M. Al-Fadel ${ }^{2} \S$ \\ ${ }^{1}$ Faculty of Science \\ Alexandria University \\ Alexandria, EGYPT \\ ${ }^{2}$ Faculty of Science \\ Omar Al-Mukhtar University \\ Al-Qubbah, LIBYA
}

\begin{abstract}
The analysis of Urysohn-Stieltjes integral operators has been studied in [1]. Here we study the existence of weakly solution of functional integral equations of Urysohn-Stieltjes type and Hammerstien-Stieltjes type in the reflexive Banach space $E$. Also, we prove the existence of the weak maximal and weak minimal solutions.
\end{abstract}

AMS Subject Classification: 26A42, 35D30, 47H30

Key Words: weak solution, Urysohn-Stieltjes integral, weak maximal and weak minimal solutions, weakly relatively compact

\section{Introduction and Preliminaries}

Consider the nonlinear Urysohn-Stieltjes integral equation

$$
x(t)=a(t)+\int_{0}^{1} f(t, s, x(s)) d_{s} g(t, s), t \in I=[0,1],
$$

where $g: I \times I \rightarrow R$ and the symbol $d_{s}$ indicates the integration with respect to $s$. Equations of type (1) and some of their generalizations were considered in paper (see [3]), for the properties of the Urysohn-Stieltjes integral (see Banaś $[1])$.

$\begin{array}{ll}\text { Received: February 11, } 2018 & \text { (C) } 2018 \text { Academic Publications }\end{array}$

${ }^{\S}$ Correspondence author 
In this paper, we study the existence of weak solutions $x \in C[I, E]$ of the Urysohn-Stieltjes functional integral equation

$$
x(t)=a(t)+\int_{0}^{1} f(t, s, x(\psi(s))) d_{s} g(t, s), \quad t \in I .
$$

As an application, we study the existence of weak solutions $x \in C[I, E]$ of the Hammerstien-Stieltjes functional integral equation

$$
x(t)=a(t)+\int_{0}^{1} k(t, s) f_{1}(s, x(\psi(s))) d_{s} g(t, s), \quad t \in I .
$$

Also, the existence of the weak maximal and weak minimal solutions will be proved.

Let $E$ be a reflexive Banach space with norm $\|$.$\| and dual E^{*}$. Denote by $C[I, E]$ the Banach space of strongly continuous functions $x: I \rightarrow E$ with sup-norm.

Now, we shall present some auxiliary results that will be need in this work. Let $E$ be a Banach space (need not be reflexive) and let $x:[a, b] \rightarrow E$, then:

(1-) $x($.$) is said to be weakly continuous (measurable) at t_{0} \in[a, b]$ if for every $\phi \in E^{*}, \phi(x()$.$) is continuous (measurable) at t_{0}$.

(2-) A function $h: E \rightarrow E$ is said to be weakly sequentially continuous if $h$ maps weakly convergent sequences in $E$ to weakly convergent sequences in $E$.

If $x$ is weakly continuous on $I$, then $x$ is strongly measurable and hence weakly measurable (see [14] and [10]). It is evident that in reflexive Banach spaces, if $x$ is weakly continuous function on $[a, b]$, then $x$ is weakly Riemann integrable (see [14]). Since the space of all weakly Riemann-Stieltjes integrable functions is not complete, we will restrict our attention to the existence of weak solutions of equation (2) in the space $C[I, E]$.

Definition 1. Let $f: I \times E \rightarrow E$. Then $f(t, u)$ is said to be weakly-weakly continuous at $\left(t_{0}, u_{0}\right)$ if given $\epsilon>0, \phi \in E^{*}$ there exists $\delta>0$ and a weakly open set $U$ containing $u_{0}$ such that

$$
\left|\phi\left(f(t, u)-f\left(t_{0}, u_{0}\right)\right)\right|<\epsilon
$$

whenever

$$
\left|t-t_{0}\right|<\delta \text { and } u \in U
$$


Now, we have the following fixed point theorem, due to O'Regan, in the reflexive Banach space (see [18]) and some propositions which will be used in the sequel (see [12]).

Theorem 2. Let $E$ be a Banach space and let $Q$ be a nonempty, bounded, closed and convex subset of $C[I, E]$ and let $F: Q \rightarrow Q$ be a weakly sequentially continuous and assume that $F Q(t)$ is relatively weakly compact in $E$ for each $t \in I$. Then, $F$ has a fixed point in the set $Q$.

Proposition 1. In reflexive Banach space, the subset is weakly relatively compact if and only if it is bounded in the norm topology.

Proposition 2. Let $E$ be a normed space with $y \in E$ and $y \neq 0$. Then there exists a $\varphi \in E^{*}$ with $\|\varphi\|=1$ and $\|y\|=\varphi(y)$.

\section{Main results}

In this section, we present our main result by proving the existence of weak solutions for equation (2) in the reflexive Banach space. Let us first state the following assumptions:

(i) $a: I \rightarrow I$ is continuous function.

(ii) $\psi: I \rightarrow I$ is continuous function such that $\psi(t) \leq t$.

(iii) $f: I \times I \times D \subset E \rightarrow E$ satisfies the following conditions:

(1) $f(., s, x(s))$ is continuous function, $\forall s \in I, x \in D \subset E$.

(2) $f(t, . .$.$) is weakly-weakly continuous function, \forall t \in I$.

(3) $\|f(t, s, x)\| \leq m(t, s)+b\|x\|, m: I \times I \rightarrow I$ is continuous function, $b$ is positive constant for $t, s \in I, x \in D$. Moreover, we put $M=$ $\max \{m(t, s): t, s \in I\}$.

(iv) The functions $t \rightarrow g(t, 1)$ and $t \rightarrow g(t, 0)$ are continuous on $I$, such that

$$
\mu=\max \left\{\sup _{t}|g(t, 1)|+\sup _{t}|g(t, 0)|, \quad t \in I\right\} .
$$

(v) For all $t_{1}, t_{2} \in I$ such that $t_{1}<t_{2}$ the function $s \rightarrow g\left(t_{2}, s\right)-g\left(t_{1}, s\right)$ is nondecreasing on $I$.

(vi) $g(0, s)=0$ for any $s \in I$. 
Remark 1. Observe that assumptions (v) and (vi) imply that the function $s \rightarrow g(t, s)$ is nondecreasing on the interval $I$, for any fixed $t \in I$ (Remark 1 in [4]). Indeed, putting $t_{2}=t, t_{1}=0$ in (v) and keeping in mind (vi), we obtain the desired conclusion. From this observation, it follows immediately that, for every $t \in I$, the function $s \rightarrow g(t, s)$ is of bounded variation on $I$.

Definition 3. By a weak solution to (2) we mean a function $x \in C[I, E]$ which satisfies the integral equation (2). This is equivalent to finding $x \in C[I, E]$ with

$$
\varphi(x(t))=\varphi\left(a(t)+\int_{0}^{1} f(t, s, x(\psi(s))) d_{s} g(t, s)\right), \quad t \in I, \forall \varphi \in E^{*} .
$$

Theorem 4. Under the assumptions (i)-(vi), the Urysohn-Stieltjes functional integral equation (2) has at least one weak solution $x \in C[I, E]$.

Proof. Define the operator $A$ by

$$
A x(t)=a(t)+\int_{0}^{1} f(t, s, x(\psi(s))) d_{s} g(t, s), \quad t \in I .
$$

For every $x \in C[I, E], f(., s, x(\psi(s)))$ is continuous on $I$, and $f(t, .,$.$) is weakly-$ weakly continuous on $I$, then $\varphi(f(t, s, x(\psi(s))))$ is continuous for every $\varphi \in$ $E^{*}, g$ is of bounded variation. Hence $f(t, s, x(\psi(s)))$ is weakly RiemannStieltjes integrable on $I$ with respect to $s \rightarrow g(t, s)$. Thus $A$ makes sense.

Now, define the set $Q_{r}$ by

$$
Q_{r}=\left\{x \in C[I, E]: \quad\|x\| \leq r, \quad r=\frac{\|a\|+M \mu}{1-b \mu}\right\} .
$$

The remainder of the proof will be given in four steps.

First, we will prove that the operator $A$ maps $C[I, E]$ into $C[I, E]$.

Let $\epsilon>0, t_{1}, t_{2} \in I, t_{2}>t_{1}$, and $t_{2}-t_{1}<\epsilon$, without loss of generality, assume that $A x\left(t_{2}\right)-A x\left(t_{1}\right) \neq 0$,

$$
\begin{aligned}
&\left\|A x\left(t_{2}\right)-A x\left(t_{1}\right)\right\| \leq\left|\varphi\left(a\left(t_{2}\right)-a\left(t_{1}\right)\right)\right| \\
&+\quad \mid \int_{0}^{1} \varphi\left(f\left(t_{2}, s, x(\psi(s))\right)\right) d_{s} g\left(t_{2}, s\right) \\
&-\int_{0}^{1} \varphi\left(f\left(t_{1}, s, x(\psi(s))\right)\right) d_{s} g\left(t_{1}, s\right) \mid \\
& \leq\left\|a\left(t_{2}\right)-a\left(t_{1}\right)\right\|+\mid \int_{0}^{1} \varphi\left(f\left(t_{2}, s, x(\psi(s))\right)\right) d_{s} g\left(t_{2}, s\right)
\end{aligned}
$$




$$
\begin{aligned}
& -\quad \int_{0}^{1} \varphi\left(f\left(t_{1}, s, x(\psi(s))\right)\right) d_{s} g\left(t_{2}, s\right) \mid \\
& +\quad \mid \int_{0}^{1} \varphi\left(f\left(t_{1}, s, x(\psi(s))\right)\right) d_{s} g\left(t_{2}, s\right) \\
& -\quad \int_{0}^{1} \varphi\left(f\left(t_{1}, s, x(s)\right)\right) d_{s} g\left(t_{1}, s\right) \mid \\
& \leq \quad\left\|a\left(t_{2}\right)-a\left(t_{1}\right)\right\| \\
& +\quad \int_{0}^{1}\left|\varphi\left(f\left(t_{2}, s, x(\psi(s))\right)-f\left(t_{1}, s, x(\psi(s))\right)\right)\right| d_{s}\left(\bigvee_{z=0}^{s} g\left(t_{2}, z\right)\right) \\
& \left.+\quad \int_{0}^{1}\left|\varphi\left(f\left(t_{1}, s, x(\psi(s))\right)\right)\right| d_{s}\left(\bigvee_{z=0}^{s}\left[g\left(t_{2}, z\right)-g\left(t_{1}, z\right)\right)\right]\right) \\
& \leq \quad\left\|a\left(t_{2}\right)-a\left(t_{1}\right)\right\|+\left\|f\left(t_{2}, s, x\right)-f\left(t_{1}, s, x\right)\right\| \int_{0}^{1} d_{s} g\left(t_{2}, s\right) \\
& +\quad \int_{0}^{1}\left\|f\left(t_{1}, s, x\right)\right\| d_{s}\left[g\left(t_{2}, s\right)-g\left(t_{1}, s\right)\right] \\
& \leq \quad\left\|a\left(t_{2}\right)-a\left(t_{1}\right)\right\|+\left\|f\left(t_{2}, s, x\right)-f\left(t_{1}, s, x\right)\right\|\left[g\left(t_{2}, 1\right)-g\left(t_{2}, 0\right)\right] \\
& \left.+\quad \int_{0}^{1} m(t, s) d_{s}\left[g\left(t_{2}, s\right)-g\left(t_{1}, s\right)\right)\right]+\int_{0}^{1} b\|x\| d_{s}\left[g\left(t_{2}, s\right)-g\left(t_{1}, s\right)\right] \\
& \leq \quad\left\|a\left(t_{2}\right)-a\left(t_{1}\right)\right\|+\left\|f\left(t_{2}, s, x\right)-f\left(t_{1}, s, x\right)\right\|\left[g\left(t_{2}, 1\right)-g\left(t_{2}, 0\right)\right] \\
& +M \int_{0}^{1} d_{s}\left[g\left(t_{2}, s\right)-g\left(t_{1}, s\right)\right]+b r \int_{0}^{1} d_{s}\left[g\left(t_{2}, s\right)-g\left(t_{1}, s\right)\right] \\
& \leq \quad\left\|a\left(t_{2}\right)-a\left(t_{1}\right)\right\|+\left\|f\left(t_{2}, s, x\right)-f\left(t_{1}, s, x\right)\right\|\left[g\left(t_{2}, 1\right)-g\left(t_{2}, 0\right)\right] \\
& +(M+b r)\left[\left(g\left(t_{2}, 1\right)-g\left(t_{1}, 1\right)\right)-\left(g\left(t_{2}, 0\right)-g\left(t_{1}, 0\right)\right)\right] \\
& \leq \quad\left\|a\left(t_{2}\right)-a\left(t_{1}\right)\right\|+\left\|f\left(t_{2}, s, x\right)-f\left(t_{1}, s, x\right)\right\|\left[g\left(t_{2}, 1\right)-g\left(t_{2}, 0\right)\right] \\
& +\quad(M+b r)\left[\left|g\left(t_{2}, 1\right)-g\left(t_{1}, 1\right)\right|+\left|g\left(t_{2}, 0\right)-g\left(t_{1}, 0\right)\right|\right] \text {. }
\end{aligned}
$$

Hence,

$$
\begin{aligned}
\| A x\left(t_{2}\right) & -A x\left(t_{1}\right)\|\leq\| a\left(t_{2}\right)-a\left(t_{1}\right) \| \\
& +\left\|f\left(t_{2}, s, x\right)-f\left(t_{1}, s, x\right)\right\|\left[g\left(t_{2}, 1\right)-g\left(t_{2}, 0\right)\right] \\
& +(M+b r)\left[\left|g\left(t_{2}, 1\right)-g\left(t_{1}, 1\right)\right|+\mid g\left(t_{2}, 0\right)-g\left(t_{1}, 0\right) \|\right],
\end{aligned}
$$

then from the continuity of the function $g$ assumption (iv) we deduce that $A$ maps $C[I, E]$ into $C[I, E]$. 
Secondly, we will prove that the operator $A$ maps $Q_{r}$ into $Q_{r}$.

Take $x \in Q_{r}$, without loss of generality assume $A x \neq 0, \quad t \in I$. By Proposition 2, we have

$$
\begin{aligned}
\|A x(t)\| & =\varphi(A x(t)) \\
& \leq|\varphi(a(t))|+\left|\varphi\left(\int_{0}^{1} f(t, s, x(\psi(s))) d_{s} g(t, s)\right)\right| \\
& \leq\|a\|+\int_{0}^{1}|\varphi(f(t, s, x(\psi(s))))| d_{s}\left(\bigvee_{z=0}^{s} g(t, z)\right) \\
& \leq\|a\|+\int_{0}^{1}\|f(t, s, x)\| d_{s}\left(\bigvee_{z=0}^{s} g(t, z)\right) \\
& \leq\|a\|+\int_{0}^{1} m(t, s) d_{s} g(t, s)+\int_{0}^{1} b\|x\| d_{s} g(t, s) \\
& \leq\|a\|+M \int_{0}^{1} d_{s} g(t, s)+b r \int_{0}^{1} d_{s} g(t, s) \\
& \leq\|a\|+M[g(t, 1)-g(t, 0)]+b r[g(t, 1)-g(t, 0)] \\
& \leq\|a\|+(M+b r)\left[\sup _{t \in I}|g(t, 1)|+\sup _{t \in I}|g(t, 0)|\right] \\
& \leq\|a\|+(M+b r) \mu .
\end{aligned}
$$

Then,

$$
\|A x(t)\| \leq\|a\|+(M+b r) \mu=r .
$$

Hence $A x \in Q_{r}$ which prove that $A: Q_{r} \rightarrow Q_{r}$ and $A Q_{r}$ is bounded in $C[I, E]$.

Thirdly, we will prove that $A Q_{r}(t)$ is relatively weakly compact in $E$.

Note that $Q_{r}$ is nonempty, uniformly bounded and strongly equicontinuous subset of $C[I, E]$, by the uniform boundedness of $A Q_{r}$, according to Proposition $1, A Q_{r}$ is relatively weakly compact.

Finally, we will prove that the operator $A$ is weakly sequentially continuous.

Let $\left\{x_{n}(t)\right\}$ be sequence in $Q_{r}$ weakly convergent to $x(t)$ in $E$, since $Q_{r}$ is closed we have $x \in Q_{r}$. Fix $t, s \in I$, since $f$ satisfies (1)-(2), then we have $f\left(t, s, x_{n}(\psi(s))\right)$ converges weakly to $f(t, s, x(\psi(s)))$. Furthermore, $(\forall \varphi \in$ $\left.E^{*}\right) \varphi\left(f\left(t, s, x_{n}(\psi(s))\right)\right)$ convergence strongly to $\varphi(f(t, s, x(\psi(s))))$.

Applying Lebesgue dominated convergence theorem,

$$
\varphi\left(\int_{0}^{1} f\left(t, s, x_{n}(\psi(s))\right) d_{s} g(t, s)\right)=\int_{0}^{1} \varphi\left(f\left(t, s, x_{n}(\psi(s))\right)\right) d_{s} g(t, s)
$$




$$
\rightarrow \int_{0}^{1} \varphi(f(t, s, x(\psi(s)))) d_{s} g(t, s), \forall \varphi \in E^{*}, t \in I,
$$

i.e. $\varphi\left(A x_{n}(t)\right) \rightarrow \varphi(A x(t)), \forall t \in I, A x_{n}(t)$ converging weakly to $A x(t)$ in $E$.

Thus, $A$ is weakly sequentially continuous on $Q_{r}$.

Since all conditions of Theorem 2 are satisfied, then the operator $A$ has at least one fixed point $x \in Q_{r}$ and the Urysohn-Stieltjes functional integral equation (2) has at least one weak solution.

\section{Hammerstien-Stieltjes function integral equation}

Consider the following assumption:

$(\text { iii })^{*}$ Let $f_{1}: I \times E \rightarrow E$ and $k: I \times I \rightarrow R_{+}$assume that $f_{1}, k$ satisfy the following assumptions:

$(1)^{*} \quad f_{1}(s, x(\psi(s)))$ is weakly-weakly continuous function.

$(2)^{*}$ There exists a continuous function $m_{1}(t)$ and constant $b>0$ such that

$$
\left\|f_{1}(t, x)\right\| \leq m_{1}(t)+b\|x\|,
$$

for $t, s \in I, x \in E$. Moreover, we put $M_{1}=\max \left\{m_{1}(t): t \in\right.$ $I\}, M_{1}>0$.

$(3)^{*} \quad k(t, s)$ is continuous function such that $K=\sup _{t}|k(t, s)|$ and $K$ is positive constant.

Definition 5. By a weak solution to (3) we mean a function $x \in C[I, E]$ which satisfies the integral equation (3). This is equivalent to finding $x \in C[I, E]$ with

$$
\varphi(x(t))=\varphi\left(a(t)+\int_{0}^{1} k(t, s) f_{1}(s, x(\psi(s))) d_{s} g(t, s)\right), t \in I \forall \varphi \in E^{*} .
$$

New for the existence of a weak solution of (3), we have the following theorem.

Theorem 6. Let the assumptions (i),(ii),(iv)-(vi) and (iii)* be satisfied. Then the Hammerstien-Stieltjes functional integral equation (3) has at least one weak solution $x \in C[I, E]$. 
Proof. Let

$$
f(t, s, x(\psi(s)))=k(t, s) f_{1}(s, x(\psi(s))) .
$$

Then from the assumption $(i i i)^{*}$, we find that the assumptions of Theorem 2 are satisfied and result follows.

\section{The weak maximal and weak minimal solutions}

Now we give the following definition.

Definition 7. Let $q(t)$ be a weak solution of (2) Then $q(t)$ is said to be a weak maximal solution of (2) if every weak solution $\mathrm{x}(\mathrm{t})$ of $(2)$ satisfies the inequality

$$
\varphi(x(t))<\varphi(q(t)), \quad \forall \varphi \in E^{*} .
$$

A weak minimal solution $s(t)$ can be defined by similar way by reversing the above inequality, i.e.

$$
\varphi(x(t))>\varphi(s(t)), \quad \forall \varphi \in E^{*}
$$

In this section, we assume that $f$ satisfies the following assumption:

(4) for any $x, y \in E$ satisfying $\varphi(x(t))<\varphi(y(t)), \quad \forall \varphi \in E^{*}$ implies that

$$
\varphi(f(t, s, x(\psi(s))))<\varphi(f(t, s, y(\psi(s)))) .
$$

Lemma 1. Let $f(t, s, x)$ satisfy assumptions of Theorem 2 and let $x(t), y(t) \in$ $C[I, E]$ on $I$ satisfy

$$
\begin{aligned}
& \varphi(x(t)) \leq \varphi(a(t))+\int_{0}^{1} \varphi(f(t, s, x(\psi(s)))) d_{s} g(t, s) \\
& \varphi(y(t)) \geq \varphi(a(t))+\int_{0}^{1} \varphi(f(t, s, x(\psi(s)))) d_{s} g(t, s)
\end{aligned}
$$

for all $\varphi \in E^{*}$, where one of them is strict.

If $(f(t, s, x))$ satisfies assumption (4), then

$$
\varphi(x(t))<\varphi(y(t)) .
$$


Proof. Let the conclusion (4) be false, then there exists $t_{1}$ such that

$$
\varphi\left(x\left(t_{1}\right)\right)=\varphi\left(y\left(t_{1}\right)\right), \quad t_{1}>0
$$

and

$$
\varphi(x(t))<\varphi(y(t)), \quad 0<t<t_{1} .
$$

Since $(f(t, s, x))$ satisfies assumption (4), we get

$$
\begin{aligned}
\varphi\left(x\left(t_{1}\right)\right) & \leq \varphi\left(g\left(t_{1}\right)\right)+\int_{0}^{1} \varphi\left(f\left(t_{1}, s, x(\psi(s))\right)\right) d_{s} g(t, s) \\
& <\varphi\left(g\left(t_{1}\right)\right)+\int_{0}^{1} \varphi\left(f\left(t_{1}, s, y(\psi(s))\right)\right) d_{s} g(t, s) \\
& <\varphi\left(y\left(t_{1}\right)\right) .
\end{aligned}
$$

Which contradicts the fact that $\varphi\left(x\left(t_{1}\right)\right)=\varphi\left(y\left(t_{1}\right)\right)$, then

$$
\varphi(x(t))<\varphi(y(t)) \text {. }
$$

Theorem 8. Let the assumptions of Theorem 2 be satisfied. If $f(t, x)$ satisfies assumption (4), then there exist a weak maximal and weak minimal solutions of (2).

Proof. First, we shall prove the existence of the weak maximal solution of (2). Let $\epsilon>0$ be given. Now consider the integral equation

$$
x_{\epsilon}(t)=a(t)+\int_{0}^{1} f_{\epsilon}\left(t, s, x_{\epsilon}(\psi(s))\right) d_{s} g(t, s),
$$

where

$$
f_{\epsilon}\left(t, s, x_{\epsilon}(\psi(s))\right)=f\left(t, s, x_{\epsilon}(\psi(s))\right)+\epsilon .
$$

Clearly the function $f_{\epsilon}\left(t, s, x_{\epsilon}\right)$ satisfies the conditions (1)-(3) of Theorem 2, and

$$
\left\|f_{\epsilon}\left(t, s, x_{\epsilon}\right)\right\| \leq m(t, s)+b\|x\|+\epsilon=m(t, s)+b_{1}\|x\| .
$$

Therefore equation (4) has a weak solution $x_{\epsilon} \in C[I, E]$ according to Theorem 2. Let $\epsilon_{1}$ and $\epsilon_{2}$ be such that $0<\epsilon_{2}<\epsilon_{1}<\epsilon$. Then,

$$
\begin{gathered}
x_{\epsilon_{1}}(t)=a(t)+\int_{0}^{1} f_{\epsilon_{1}}\left(t, s, x_{\epsilon_{1}}(\psi(s))\right) d_{s} g(t, s) \\
x_{\epsilon_{1}}(t)=a(t)+\int_{0}^{1}\left(f\left(t, s, x_{\epsilon_{1}}(\psi(s))\right)+\epsilon_{1}\right) d_{s} g(t, s)
\end{gathered}
$$

implies that 


$$
\begin{aligned}
& \varphi\left(x_{\epsilon_{1}}(t)\right)>\varphi(a(t))+\int_{0}^{1} \varphi\left(f\left(t, s, x_{\epsilon_{1}}(\psi(s))\right)\right)+\epsilon_{2} d_{s} g(t, s), \\
& \varphi\left(x_{\epsilon_{2}}(t)\right)=\varphi(a(t))+\int_{0}^{1} \varphi\left(f\left(t, s, x_{\epsilon_{2}}(\psi(s))\right)\right)+\epsilon_{2} d_{s} g(t, s) .
\end{aligned}
$$

Using Lemma 1, then (5) and (6) imply

$$
\varphi\left(x_{\epsilon_{2}}(t)\right)<\varphi\left(x_{\epsilon_{1}}(t)\right), \quad t \in[0,1] .
$$

As shown before in the proof of Theorem 2, the family of functions $x(t)$ defined by (4) is uniformly bounded and of strongly equicontinuous functions. Hence by the Arzela-Ascoli Theorem, there exists a decreasing sequence $\epsilon_{n}$ such that $\epsilon \rightarrow 0$ as $n \rightarrow \infty$ and $\lim _{n \rightarrow \infty} x_{\epsilon_{n}}(t)$ exists uniformly in $[0,1]$ and denote this limit by $q(t)$. From the weakly continuity of the function $f_{\epsilon_{n}}$ and applying the Lebesgue Dominated Convergence Theorem, we get

$$
q(t)=\lim _{n \rightarrow \infty} x_{\epsilon_{n}}(t)=a(t)+\int_{0}^{1}(f(t, s, q(\psi(s)))) d_{s} g(t, s),
$$

which proves that $q(t)$ as a solution of (2).

Finally, we shall show that $q(t)$ is the weak maximal solution of (2). To do this, let $x(t)$ be any weak solution of (2). Then,

$$
\begin{aligned}
\varphi\left(x_{\epsilon}(t)\right) & =\varphi(a(t))+\int_{0}^{1}\left(\varphi\left(f\left(t, s, x_{\epsilon}(\psi(s))\right)\right)+\epsilon\right) d_{s} g(t, s) \\
& >\varphi(a(t))+\int_{0}^{1} \varphi\left(f\left(t, s, x_{\epsilon}(\psi(s))\right)\right) d_{s} g(t, s),
\end{aligned}
$$

and

$$
\varphi(x(t))=\varphi(a(t))+\int_{0}^{1}\left(\varphi(f(t, s, x(\psi(s)))) d_{s} g(t, s),\right.
$$

and applying Lemma 1, we get

$$
\varphi\left(x_{\epsilon}(t)\right)>\varphi(x(t)) .
$$

From the uniqueness of the maximal solution (see [10]), it is clear that $x_{\epsilon}(t)$ tends to $q(t)$ uniformly in $t \in[0,1]$ as $\epsilon \rightarrow 0$.

By similar way as above, we can prove that $s(t)$ is the weak minimal solution of (2).

The weak maximal and minimal solutions of (3) can be defined in the same fashion as done above.

Now, the function $f_{1}$ is assumed to satisfy the following assumption: 
$\left(4^{*}\right)$ for any $x, y \in E$ satisfying $\varphi(x(t))<\varphi(y(t)), \quad \forall \varphi \in E^{*}$ implies that

$$
\varphi\left(f_{1}(s, x(\psi(s)))\right)<\varphi\left(f_{1}(s, y(\psi(s)))\right) .
$$

Now the following lemma can be proved.

Lemma 2. Let $f_{1}(t, x), k(t, s)$ satisfy the assumptions of Theorem 3 and let $x(t), y(t) \in C[I, E]$ on $I$ satisfy

$$
\begin{aligned}
& \varphi(x(t)) \leq \varphi(a(t))+\int_{0}^{1} k(t, s) \varphi\left(f_{1}(s, x(\psi(s)))\right) d_{s} g(t, s), \\
& \varphi(y(t)) \geq \varphi(a(t))+\int_{0}^{1} k(t, s) \varphi\left(f_{1}(s, x(\psi(s)))\right) d_{s} g(t, s),
\end{aligned}
$$

where one of these is strict. If $f_{1}(t, x)$ satisfies assumption $\left(4^{*}\right)$. Then,

$$
\varphi(x(t))<\varphi(y(t)) \text {. }
$$

Theorem 9. Let the assumptions of Theorem 3 be satisfied. If $f_{1}(t, x)$ satisfies assumption $\left(4^{*}\right)$, then there exist a weak maximal and weak minimal solutions of (3).

Proof. First, we shall prove the existence of the weak maximal solution of (3). Let $\epsilon>0$ be given. Now consider the integral equation

$$
x_{\epsilon}(t)=a(t)+\int_{0}^{1} k(t, s) f_{1 \epsilon}\left(s, x_{\epsilon}(\psi(s))\right) d_{s} g(t, s),
$$

where

$$
f_{1_{\epsilon}}\left(s, x_{\epsilon}(s)\right)=f_{1}\left(s, x_{\epsilon}(\psi(s))\right)+\epsilon .
$$

Clearly the function $f_{1_{\epsilon}}\left(s, x_{\epsilon}\right)$ satisfies the conditions $(1)^{*},(2)^{*}$ of Theorem 3 , and

$$
\left\|f_{1_{\epsilon}}\left(s, x_{\epsilon}\right)\right\| \leq m_{1}(t)+b\|x\|+\epsilon=m_{1}(t)+b_{1}\|x\| .
$$

Therefore equation (7) has a weak solution $x_{\epsilon} \in C[I, E]$ according to Theorem 3. Let $\epsilon_{1}$ and $\epsilon_{2}$ be such that $0<\epsilon_{2}<\epsilon_{1}<\epsilon$. Then,

$$
\begin{gathered}
x_{\epsilon_{1}}(t)=a(t)+\int_{0}^{1} k(t, s) f_{1_{\epsilon_{1}}}\left(s, x_{\epsilon_{1}}(\psi(s))\right) d_{s} g(t, s), \\
x_{\epsilon_{1}}(t)=a(t)+\int_{0}^{1} k(t, s)\left(f_{1}\left(s, x_{\epsilon_{1}}(\psi(s))\right)+\epsilon_{1}\right) d_{s} g(t, s),
\end{gathered}
$$

implies that 


$$
\begin{aligned}
& \varphi\left(x_{\epsilon_{1}}(t)\right)>\varphi(a(t))+\int_{0}^{1} k(t, s) \varphi\left(f_{1}\left(s, x_{\epsilon_{1}}(\psi(s))\right)\right)+\epsilon_{2} d_{s} g(t, s), \\
& \varphi\left(x_{\epsilon_{2}}(t)\right)=\varphi(a(t))+\int_{0}^{1} k(t, s) \varphi\left(f_{1}\left(s, x_{\epsilon_{2}}(\psi(s))\right)\right)+\epsilon_{2} d_{s} g(t, s) .
\end{aligned}
$$

Using Lemma 2, then (8) and (9) implies

$$
\varphi\left(x_{\epsilon_{2}}(t)\right)<\varphi\left(x_{\epsilon_{1}}(t)\right), \quad t \in[0,1] .
$$

As shown before in the proof of Theorem 3 , the family of functions $x_{\epsilon}(t)$ defined by (7) is uniformly bounded and of strongly equicontinuous functions. Hence by the Arzela-Ascoli Theorem, there exists a decreasing sequence $\epsilon_{n}$ such that $\epsilon \rightarrow 0$ as $n \rightarrow \infty$ and $\lim _{n \rightarrow \infty} x_{\epsilon_{n}}(t)$ exists uniformly in $[0,1]$ and denote this limit by $q(t)$. From the weakly continuity of the function $f_{1_{\epsilon_{n}}}$ and applying Lebesgue Dominated Convergence Theorem, we get

$$
q(t)=\lim _{n \rightarrow \infty} x_{\epsilon_{n}}(t)=a(t)+\int_{0}^{1} k(t, s) f_{1}(s, q(\psi(s))) d_{s} g(t, s),
$$

which proves that $q(t)$ as a solution of (3).

Finally, we shall show that $q(t)$ is the weak maximal solution of (3). To do this, let $x(t)$ be any weak solution of (3). Then

$$
\begin{aligned}
\varphi\left(x_{\epsilon}(t)\right) & =\varphi(a(t))+\int_{0}^{1} k(t, s)\left(\varphi\left(f_{1}\left(s, x_{\epsilon}(\psi(s))\right)\right)+\epsilon\right) d_{s} g(t, s) \\
& >\varphi(a(t))+\int_{0}^{1} k(t, s) \varphi\left(f_{1}\left(s, x_{\epsilon}(\psi(s))\right)\right) d_{s} g(t, s),
\end{aligned}
$$

and

$$
\varphi(x(t))=\varphi(a(t))+\int_{0}^{1} k(t, s) \varphi\left(f_{1}(s, x(\psi(s)))\right) d_{s} g(t, s),
$$

applying Lemma 2, we get

$$
\varphi\left(x_{\epsilon}(t)\right)>\varphi(x(t))
$$

from the uniqueness of the maximal solution (see [10]), it is clear that $x_{\epsilon}(t)$ tends to $q(t)$ uniformly in $t \in[0,1]$ as $\epsilon \rightarrow 0$.

By similar way as done above we can prove that $s(t)$ is the weak minimal solution of (3).

In what follows, we provide some examples illustrating the above obtained results. 
Example 1. Consider the function $g: I \times I \rightarrow R$ defined by the formula

$$
g(t, s)=t^{3}+t s, \quad t \in I
$$

It can be easily seen that the function $g(t, s)$ satisfy assumptions (iv)-(vi) given in Theorem 2. In this case, the Urysohn-Stieltjes functional integral equation (2) has the form

$$
\left.x(t)=a(t)+\int_{0}^{1} t f(t, s, x(\psi(s)))\right) d s, \quad t \in I .
$$

Therefore, the equation (10) has at least one weak solution $x \in C[I, E]$, if the functions $a, \psi$ and $f$ satisfy the assumptions (i)-(iii).

Example 2. Consider the function $g: I \times I \rightarrow R$ defined by the formula

$$
g(t, s)= \begin{cases}t \ln \frac{t+s}{t}, & \text { for } t \in(0,1], \quad s \in I, \\ 0, & \text { for } t=0, \quad s \in I .\end{cases}
$$

Also, the function $g(t, s)$ satisfy assumptions (iv)-(vi) given in Theorem 2. In this case, the Urysohn-Stieltjes functional integral equation (2) has the form

$$
\left.x(t)=a(t)+\int_{0}^{1} \frac{t}{t+s} f(t, s, x(\psi(s)))\right) d s, \quad t \in I .
$$

Therefore, the equation (11) has at least one weak solution $x \in C[I, E]$, if the functions $a, \psi$ and $f$ satisfy the assumptions (i)-(iii).

\section{Acknowledgment}

The authors is grateful to the referee for a number of helpful suggestions to improve the paper.

\section{References}

[1] J. Banaś, Some properties of Urysohn-Stieltjes integral operators, Intern. J. Math. and Math. Sci., 21 (1998), 78-88.

[2] J. Banaś and J. Dronka, Integral operators of Volterra-Stieltjes type, their properties and applications, Math. Comput. Modelling, 32, No 1113 (2000), 1321-1331.

[3] J. Banaś, J.C. Mena, Some properties of nonlinear Volterra-Stieltjes integral operators, Comput. Math. Appl., 49 (2005), 1565-1573. 
[4] J. Banaś, D. O’Regan, Volterra-Stieltjes integral operators, Math. Comput. Modelling, 41 (2005), 335-344.

[5] J. Banaś, J.R. Rodriguez and K. Sadarangani, On a class of UrysohnStieltjes quadratic integral equations and their applications, J. Comput. Appl. Math., I13 (2000), 35-50.

[6] J. Banaś and K. Sadarangani, Solvability of Volterra-Stieltjes operatorintegral equations and their applications, Comput. Math. Appl., 41, No 12 (2001), 1535-1544.

[7] C.W. Bitzer, Stieltjes-Volterra integral equations, Illinois J. Math., 14 (1970), 434-451.

[8] S. Chen, Q. Huang and L.H. Erbe, Bounded and zero-convergent solutions of a class of Stieltjes integro-differential equations, Proc. Amer. Math. Soc., 113 (1991), 999-1008.

[9] J. Diestel, J.J. Uhl Jr., Vector Measures, Ser. Math. Surveys, Vol. 15, Amer. Math. Soc., Providence, RI (1977).

[10] N. Dunford, J.T. Schwartz, Linear Operators, Interscience, Wiley, New York (1958).

[11] A.M.A. EL-Sayed, W.G. El-Sayed, A.A.H. Abd El-Mowla, VolterraStieltjes integral equation in refexive Banach space, Electronic J. of Math. Anal. and Appl., 5, No 1 (2017), 287-293.

[12] A.M.A. EL-Sayed, H.H.G. Hashem, Weak maximal and minimal solutions for Hammerstein and Urysohn integral equations in reflexive Banach spaces, Differential Equations and Control Processes, 4 (2008), 50-62.

[13] R.F. Geitz, Pettis integration, Proc. Amer. Math. Soc., 82 (1981), 81-86.

[14] E. Hille and R.S. Phillips, Functional Analysis and Semi-Groups, Amer. Math. Soc. Colloq. Publ. Providence, R.I. (1957).

[15] J.S. Macnerney, Integral equations and semigroups, Illinois J. Math., 7 (1963), 148-173.

[16] A.B. Mingarelli, Volterra-Stieltjes Integral Equations And Generalized Ordinary Differential Expressions, Lecture Notes in Math., Vol. 989, Springer (1983). 
[17] I.P. Natanson, Theory of Functions of a Real Variable, Ungar, New York (1960).

[18] D. O'Regan, Fixed point theory for weakly sequentially continuous mapping, Math. Comput. Modeling, 27 (1998), 1-14. 
